\title{
BSM Developments and Tools
}

\author{
Tomás E. Gonzalo ${ }^{a, *}$ \\ ${ }^{a}$ Institute for Theoretical Particle Physics and Cosmology (TTK), RWTH Aachen University, D-52056 \\ Aachen, Germany \\ E-mail: gonzalo@physik.rwth-aachen.de
}

In this conference paper I introduce a selection of BSM tools and describe their most recent developments. I choose to focus on tools for the reinterpretation of LHC searches, tools that compute dark matter constraints, beyond-the-Standard-Model (BSM) inference tools, and tools that calculate amplitudes and cross sections directly from the Lagrangian.

The Ninth Annual Conference on Large Hadron Collider Physics - LHCP2021

7-12 June 2021

Online

${ }^{*}$ Speaker

(C) Copyright owned by the author(s) under the terms of the Creative Commons 


\section{Introduction}

We are spoiled for choices when it comes to BSM tools. For many years the community has developed tools for every aspect of BSM physics, from various computations of observables, to abstract level tools able to handle the full Lagrangian of a theory. Due to the large amount of software available, in this conference article I will only describe a selection of tools and their recent developments. I will focus on tools for the recasting of LHC searches, dark matter (DM) tools, statistical inference tools and Lagrangian-level tools.

\section{Reinterpretation of $\mathrm{LHC}$ searches}

The reinterpretation of BSM searches has been a profilic field of study in particle physics. Significant advances have been made in the last few years in the interaction between the experimental and theoretical communities, and the exchange of information between them is at its highest, especially after the release of simplified and full likelihoods by CMS and ATLAS, respectively [1]. This has had profound positive effects on the whole community as well as the physics outcomes, as it has helped with the long-term preservation of the results and provides motivation for the design of new analyses. Consequently, many recasting tools have been developed, following two different approaches: the Simplified Models approach, or the Fast Simulation approach.

The Simplified Models approach tackles the issue of recasting by decomposing a given model into simplified model topologies and comparing the predicted cross section times branching ratio with that reported by the experiments [2]. As it does not require any simulation of collisions, this approach is extremely fast and perfectly suitable for large parameter fits. The prominent tool that implements this approach is SModelS, which is in fact responsible for pioneering the idea [2]. SModelS currently contains the largest database of implemented analyses in the community, with over 100 run I and II available analyses. With its latest version, SModelS can be interfaced with the full likelihoods provided by the ATLAS experiment [3]. Another notable tool that implements the Simplified Model approach is DarkCAST, a recasting tool for dark photon searches [4].

On the other hand is the Fast Simulation approach, which requires to perform a Monte-Carlo simulation of the hard scattering process first, then drag the generated events through some form of detector modelling and lastly pass them through an event analysis framework. There are many available event analysis frameworks, some of them with native detector modelling. A non-exhaustive list of these include MadAnalysis 5 [7-12], which can be used with a detector simulation or transfer functions and contains around 40 run I and II analyses; CheckMATE [13, 14], which contains over 50 run I and II analyses, and was recently extended to support long-lived particle searches in addition to prompt particle searches; ColliderBit [15], which includes its own detector modelling in the form fast 4-vector smearing (Buckfast), and has a database of around 40 analyses, from runs I and II $^{1}$; and Rivet [17, 18], which has currently "only" 30 BSM analyses, but boasts a huge library of SM analyses, upwards of 800. In fact, it has been shown that SM inclusive measurements also set strong constraints on new physics, and the tool Contur has been developed for this purpose [19-23]. A comparison of the performance of these tools for a specific CMS search for supersymmetric (SUSY) particles [24] was performed [25] and strong agreement was seen across all included tools.

\footnotetext{
${ }^{1}$ ColliderBit is part of the GAMBIT framework, but it will soon be released as a standalone package
} 


\section{Dark Matter}

Searches for DM are a strong component of the physics agenda of the LHC experiments, but the strongest constraints on DM models arise often from direct and indirect detection, as well as from the relic density (RD) of DM, and thus many tools have been developed over the years for that purpose $^{2}$. In recent times, there is a tendency towards the development of model-independent tools, able to constrain a large variety of BSM models. In addition, many existing and new tools attempt a comprehensive approach to constraints of DM, intending to simultaneously provide constraints from RD, direct and indirect detection. The most employed comprehensive tools developed by the community are DarkSUSY 6 [27, 28], recently expanded to compute the RD beyond the standard kinetic equilibrium; MicrOMEGAs [29-35], whose most recent upgrade included computations of freeze-in RD; MadDM [36-39], part of the MadGraph toolchain; and SuperISO Relic [40-42], which is widely used for SUSY models.

Even though comprehensive tools can cover all aspects of DM constraints, some more dedicated tools are better suited to compute a single constraint with higher accuracy. In the case of constraints from direct detection (DD), the front runner is DDCalc [43,44], which contains the largest collection of DD experiments to date, and can be used for complete as well as effective DM models, with RG evolution provided by DirectDM $[45,46]$. In the indirect direction frontier the amount of available tools is too large to recount here. A short list of selected tools include Capt'n General [47], which imposes constraints on DM capture in the Sun, nulike [48, 49], which calculates constraints from the DM annihilation to neutrinos, and gamLike [43], which deals with DM annihilation to $\gamma$-rays. Lastly one can impose constraints on DM models from cosmological observations. In particular, the energy injected into the Cosmic Microwave Background from DM annihilations in the early Universe can be computed with DarkAges [50], part of the ExoCLASS branch of CLASS [51].

\section{Inference and Sampling}

It has long been the goal of many particle theorists to assess the validity of BSM models in light of data. However, the vast quantities of data produced by the experiments, and the increasingly large parameter spaces of the models, makes this a difficult task. The common practices of overlaying exclusing regions and sampling the parameter space using random or grid samplers are insufficient and much information is lost along the way. Composite likelihoods that keep all the information about experimental data, smart sampling strategies to explore large and intricate parameter spaces [52], and a rigorous treatment of statistics are critical features that significantly improve and simplify this task [53]. And for this purpose many global fitting tools have been developed that include those features and that are targeted, though not exclusively, to BSM models.

Bayesian inference has been a staple in astrophysics for many years, and there are many public tools developed for that purpose, but in the field of BSM phenomenology the tendency has been to develop private fitters for specific purposes, e.g. flavour fits, neutrino oscillations, SUSY, etc. Only in recent years various public inference tools have been released. The first "wave" of publicly available global fitters, such as Fittino [54], SuperBayes [55] or EasyScanHEP [56], were developed to fit various SUSY models to LHC data, and some are still in use today.

\footnotetext{
${ }^{2} \mathrm{~A}$ thorough review of many DM tools can be found in [26]
} 
The revolution in the field of BSM inference came with the advent of model-independent tools. The front-runner for SM fits is HEPFit [57], built upon a Bayesian Markov chain Monte Carlo, which includes a vast library of electroweak precision, Higgs and flavour observables, and can also work with BSM models $[58,59]$. Nevertheless, the indisputable leader of BSM inference studies is the GAMBIT tool [16, 60]. GAMBIT contains a huge library of likelihoods and observables for collider physics [15], dark matter [43], flavour [61], precision observables [62], neutrino physics [63] and cosmology [64], as well as an extensive database of models and sampling strategies, both frequentist and Bayesian [65]. GAMBIT has been employed in studies of a large variety of BSM modes, including SUSY models [66-68], Higgs-portal DM models [44, 69, 70], EFT DM models [71], axion and axion-like particle models [72, 73], models with right-handed neutrinos [63], fits of neutral flavour anomalies [74] and cosmological fits of neutrino masses [75].

\section{Lagrangians and Model Building}

In addition to the software developed for the computation of observables, there is a small collection of tools that go one step further, and attempt to bridge the gap between the full Lagrangian of the BSM theory and the required amplitudes and cross sections. Some of these tools, such as FeynRules [76, 77], SARAH [78-80] and LanHEP [81-83], compute the Feynman rules directly from the Lagrangian, while others, such as FeynArts/FormCalc [84-86] or CalcHEP [87], use those to compute the required amplitudes. Although widely used, the main disadvantage of most of these tools is that they are written in Mathematica, which is a proprietary software framework and it is notably slower than other interpreted or compiled languages. Very recently a new tool has been released, MARTY [88], a fully open-source framework that uses symbolic computations in C ++ to obtain amplitudes and cross sections from the Lagrangian for any BSM theory. Finally, the last step in the BSM toolchain is to automatically connect the amplitudes, cross sections, etc, obtained by the Lagrangian-level tools, with the calculation of likelihoods and observables. This step is most often done by hand, by manually embedding the code or expressions outputted by the above tools where needed. Fortunately, an automatic integration of this step can now be achieved with GUM [89]. By interfacing with SARAH and FeynRules, GUM extracts all information about particles, parameters and expressions, auto-generates code in GAMBIT, and uses it to impose DM, collider and vacuum stability constraints. Using both GUM and GAMBIT one can start with a Lagrangian and end with a complete likelihood analysis without writing a single line of code.

\section{Summary}

In this conference article I have introduced a selection of tools for BSM physics and described their most recent developments. The state of the communication between the theory and experimental communities is currently at its best, and that improves the quality and relevance of the results from recasting tools. There is a community trend towards the development of model-independent tools, as it is the case for some of the DM comprehensive tools, as well as the leading global fitting frameworks, HEPFit and GAMBIT. And lastly, with MARTY and GUM we have managed to complete the BSM toolchain, from Lagrangian to likelihoods, using only open-source software. 


\section{References}

[1] LHC Reinterpretation Forum collaboration, W. Abdallah et al., Reinterpretation of LHC Results for New Physics: Status and Recommendations after Run 2, SciPost Phys. 9 (2020) 022, [2003.07868].

[2] S. Kraml, S. Kulkarni, U. Laa, A. Lessa, W. Magerl, D. Proschofsky-Spindler et al., SModelS: a tool for interpreting simplified-model results from the LHC and its application to supersymmetry, Eur. Phys. J. C 74 (2014) 2868, [1312.4175].

[3] G. Alguero, S. Kraml and W. Waltenberger, A SModelS interface for pyhf likelihoods, Comput. Phys. Commun. 264 (2021) 107909, [2009.01809].

[4] P. Ilten, Y. Soreq, M. Williams and W. Xue, Serendipity in dark photon searches, JHEP 06 (2018) 004, [1801.04847].

[5] T. Sjostrand, S. Mrenna and P. Z. Skands, A Brief Introduction to PYTHIA 8.1, Comput. Phys. Commun. 178 (2008) 852-867, [0710.3820].

[6] DELPHES 3 collaboration, J. de Favereau, C. Delaere, P. Demin, A. Giammanco, V. Lemaître, A. Mertens et al., DELPHES 3, A modular framework for fast simulation of a generic collider experiment, JHEP 02 (2014) 057, [1307.6346].

[7] E. Conte, B. Fuks and G. Serret, MadAnalysis 5, A User-Friendly Framework for Collider Phenomenology, Comput. Phys. Commun. 184 (2013) 222-256, [1206.1599].

[8] E. Conte, B. Dumont, B. Fuks and C. Wymant, Designing and recasting LHC analyses with MadAnalysis 5, Eur. Phys. J. C 74 (2014) 3103, [1405.3982].

[9] B. Dumont, B. Fuks, S. Kraml, S. Bein, G. Chalons, E. Conte et al., Toward a public analysis database for LHC new physics searches using MADANALYSIS 5, Eur. Phys. J. C 75 (2015) 56, [1407.3278].

[10] E. Conte and B. Fuks, Confronting new physics theories to LHC data with MADANALYSIS 5, Int. J. Mod. Phys. A 33 (2018) 1830027, [1808.00480].

[11] J. Y. Araz, M. Frank and B. Fuks, Reinterpreting the results of the LHC with MadAnalysis 5: uncertainties and higher-luminosity estimates, Eur. Phys. J. C 80 (2020) 531, [1910 . 11418].

[12] J. Y. Araz, B. Fuks and G. Polykratis, Simplified fast detector simulation in MADANALYSIS 5, Eur. Phys. J. C 81 (2021) 329, [2006.09387].

[13] M. Drees, H. Dreiner, D. Schmeier, J. Tattersall and J. S. Kim, CheckMATE: Confronting your Favourite New Physics Model with LHC Data, Comput. Phys. Commun. 187 (2015) 227-265, [1312.2591].

[14] D. Dercks, N. Desai, J. S. Kim, K. Rolbiecki, J. Tattersall and T. Weber, CheckMATE 2: From the model to the limit, Comput. Phys. Commun. 221 (2017) 383-418, [1611.09856]. 
[15] GAMBIT collaboration, C. Balázs et al., ColliderBit: a GAMBIT module for the calculation of high-energy collider observables and likelihoods, Eur. Phys. J. C 77 (2017) 795, [1705.07919].

[16] GAMBIT collaboration, P. Athron et al., GAMBIT: The Global and Modular Beyond-the-Standard-Model Inference Tool, Eur. Phys. J. C 77 (2017) 784, [1705. 07908].

[17] A. Buckley, J. Butterworth, D. Grellscheid, H. Hoeth, L. Lonnblad, J. Monk et al., Rivet user manual, Comput. Phys. Commun. 184 (2013) 2803-2819, [1003. 0694].

[18] C. Bierlich et al., Robust Independent Validation of Experiment and Theory: Rivet version 3, SciPost Phys. 8 (2020) 026, [1912.05451].

[19] J. M. Butterworth, D. Grellscheid, M. Krämer, B. Sarrazin and D. Yallup, Constraining new physics with collider measurements of Standard Model signatures, JHEP 03 (2017) 078, [1606.05296].

[20] S. Amrith, J. M. Butterworth, F. F. Deppisch, W. Liu, A. Varma and D. Yallup, LHC Constraints on a B - L Gauge Model using Contur, JHEP 05 (2019) 154, [1811.11452].

[21] A. Buckley, J. M. Butterworth, L. Corpe, D. Huang and P. Sun, New sensitivity of current LHC measurements to vector-like quarks, SciPost Phys. 9 (2020) 069, [2006. 07172].

[22] J. M. Butterworth, M. Habedank, P. Pani and A. Vaitkus, A study of collider signatures for two Higgs doublet models with a Pseudoscalar mediator to Dark Matter, SciPost Phys. Core 4 (2021) 003, [2009.02220].

[23] A. Buckley et al., Testing new physics models with global comparisons to collider measurements: the Contur toolkit, SciPost Phys. Core 4 (2021) 013, [2102. 04377].

[24] CMS collaboration, A. M. Sirunyan et al., Search for new physics in events with two soft oppositely charged leptons and missing transverse momentum in proton-proton collisions at $\sqrt{s}=13$ TeV, Phys. Lett. B 782 (2018) 440-467, [1801.01846].

[25] G. Brooijmans et al., Les Houches 2019 Physics at TeV Colliders: New Physics Working Group Report, in 11th Les Houches Workshop on Physics at TeV Colliders: PhysTeV Les Houches, 2, 2020, 2002.12220.

[26] C. Arina, Review on Dark Matter Tools, PoS TOOLS2020 (2021) 001, [2012.09462].

[27] P. Gondolo, J. Edsjo, P. Ullio, L. Bergstrom, M. Schelke and E. A. Baltz, DarkSUSY: Computing supersymmetric dark matter properties numerically, JCAP 07 (2004) 008, [astro-ph/0406204].

[28] T. Bringmann, J. Edsjö, P. Gondolo, P. Ullio and L. Bergström, DarkSUSY 6 : An Advanced Tool to Compute Dark Matter Properties Numerically, JCAP 07 (2018) 033, [1802 . 03399]. 
[29] G. Belanger, F. Boudjema, A. Pukhov and A. Semenov, MicrOMEGAs: A Program for calculating the relic density in the MSSM, Comput. Phys. Commun. 149 (2002) 103-120, [hep-ph/0112278].

[30] G. Belanger, F. Boudjema, A. Pukhov and A. Semenov, micrOMEGAs: Version 1.3, Comput. Phys. Commun. 174 (2006) 577-604, [hep-ph/0405253].

[31] G. Belanger, F. Boudjema, A. Pukhov and A. Semenov, MicrOMEGAs 2.0: A Program to calculate the relic density of dark matter in a generic model, Comput. Phys. Commun. 176 (2007) 367-382, [hep-ph/0607059].

[32] G. Belanger, F. Boudjema, A. Pukhov and A. Semenov, Dark matter direct detection rate in a generic model with micrOMEGAs 2.2, Comput. Phys. Commun. 180 (2009) 747-767, [0803.2360].

[33] G. Belanger, F. Boudjema, P. Brun, A. Pukhov, S. Rosier-Lees, P. Salati et al., Indirect search for dark matter with micrOMEGAs2.4, Comput. Phys. Commun. 182 (2011) 842-856, [1004.1092].

[34] G. Belanger, F. Boudjema, A. Pukhov and A. Semenov, micrOMEGAs_3: A program for calculating dark matter observables, Comput. Phys. Commun. 185 (2014) 960-985, [1305.0237].

[35] G. Bélanger, F. Boudjema, A. Pukhov and A. Semenov, micrOMEGAs4.1: two dark matter candidates, Comput. Phys. Commun. 192 (2015) 322-329, [1407.6129].

[36] M. Backovic, K. Kong and M. McCaskey, MadDM v.1.0: Computation of Dark Matter Relic Abundance Using MadGraph5, Physics of the Dark Universe 5-6 (2014) 18-28, [1308.4955].

[37] M. Backović, A. Martini, O. Mattelaer, K. Kong and G. Mohlabeng, Direct Detection of Dark Matter with MadDM v.2.0, Phys. Dark Univ. 9-10 (5, 2015) 37-50, [1505. 04190].

[38] F. Ambrogi, C. Arina, M. Backovic, J. Heisig, F. Maltoni, L. Mantani et al., MadDM v.3.0: a Comprehensive Tool for Dark Matter Studies, Phys. Dark Univ. 24 (2019) 100249, [1804.00044].

[39] C. Arina, J. Heisig, F. Maltoni, L. Mantani, D. Massaro, O. Mattelaer et al., Studying dark matter with MadDM 3.1: a short user guide, PoS TOOLS2020 (2021) 009, [2012.09016].

[40] A. Arbey and F. Mahmoudi, SuperIso Relic: A Program for calculating relic density and flavor physics observables in Supersymmetry, Comput. Phys. Commun. 181 (2010) 1277-1292, [0906.0369].

[41] A. Arbey and F. Mahmoudi, SuperIso Relic v3.0: A program for calculating relic density and flavour physics observables: Extension to NMSSM, Comput. Phys. Commun. 182 (2011) 1582-1583. 
[42] A. Arbey, F. Mahmoudi and G. Robbins, SuperIso Relic v4: A program for calculating dark matter and flavour physics observables in Supersymmetry, Comput. Phys. Commun. 239 (2019) 238-264, [1806.11489].

[43] GAMBIT Dark Matter Workgroup collaboration, T. Bringmann et al., DarkBit: A GAMBIT module for computing dark matter observables and likelihoods, Eur. Phys. J. C 77 (2017) 831, [1705.07920].

[44] GAMBIT collaboration, P. Athron et al., Global analyses of Higgs portal singlet dark matter models using GAMBIT, Eur. Phys. J. C 79 (2019) 38, [1808.10465].

[45] F. Bishara, J. Brod, B. Grinstein and J. Zupan, DirectDM: a tool for dark matter direct detection, 1708.02678.

[46] J. Brod, A. Gootjes-Dreesbach, M. Tammaro and J. Zupan, Effective Field Theory for Dark Matter Direct Detection up to Dimension Seven, JHEP 10 (2018) 065, [1710 . 10218].

[47] N. A. Kozar, A. Caddell, L. Fraser-Leach, P. Scott and A. C. Vincent, Capt'n General: A generalized stellar dark matter capture and heat transport code, in Tools for High Energy Physics and Cosmology, 5, 2021, 2105.06810.

[48] IceCube collaboration, P. Scott et al., Use of event-level neutrino telescope data in global fits for theories of new physics, JCAP 11 (2012) 057, [1207. 0810].

[49] ICECube collaboration, M. G. Aartsen et al., Improved limits on dark matter annihilation in the Sun with the 79-string IceCube detector and implications for supersymmetry, JCAP 04 (2016) 022, [1601.00653].

[50] P. Stöcker, M. Krämer, J. Lesgourgues and V. Poulin, Exotic energy injection with ExoCLASS: Application to the Higgs portal model and evaporating black holes, JCAP $\mathbf{0 3}$ (2018) 018, [1801.01871].

[51] D. Blas, J. Lesgourgues and T. Tram, The Cosmic Linear Anisotropy Solving System (CLASS). Part II: Approximation schemes, JCAP 2011 (July, 2011) 034, [1104. 2933].

[52] DarkMachines High Dimensional Sampling Group collaboration, C. Balázs et al., A comparison of optimisation algorithms for high-dimensional particle and astrophysics applications, JHEP 05 (2021) 108, [2101.04525].

[53] S. S. AbdusSalam et al., Simple and statistically sound strategies for analysing physical theories, 2012.09874.

[54] P. Bechtle et al., Constrained Supersymmetry after two years of LHC data: a global view with Fittino, JHEP 06 (2012) 098, [1204 .4199].

[55] R. Ruiz de Austri, R. Trotta and L. Roszkowski, A Markov chain Monte Carlo analysis of the CMSSM, JHEP 05 (2006) 002, [hep-ph/0602028]. 
[56] C. Han, K.-i. Hikasa, L. Wu, J. M. Yang and Y. Zhang, Status of CMSSM in light of current LHC Run-2 and LUX data, Phys. Lett. B 769 (2017) 470-476, [1612.02296].

[57] J. De Blas et al., HEPfit: a code for the combination of indirect and direct constraints on high energy physics models, Eur. Phys. J. C 80 (2020) 456, [1910.14012].

[58] C.-W. Chiang, G. Cottin and O. Eberhardt, Global fits in the Georgi-Machacek model, Phys. Rev. D 99 (2019) 015001, [1807.10660].

[59] D. Chowdhury and O. Eberhardt, Update of Global Two-Higgs-Doublet Model Fits, JHEP 05 (2018) 161, [1711.02095].

[60] A. Kvellestad, P. Scott and M. White, GAMBIT and its Application in the Search for Physics Beyond the Standard Model, 1912.04079.

[61] GAMBIT Flavour Workgroup collaboration, F. U. Bernlochner et al., FlavBit: A GAMBIT module for computing flavour observables and likelihoods, Eur. Phys. J. C 77 (2017) 786, [1705.07933].

[62] GAMBIT Models Workgroup collaboration, P. Athron et al., SpecBit, DecayBit and PrecisionBit: GAMBIT modules for computing mass spectra, particle decay rates and precision observables, Eur. Phys. J. C 78 (2018) 22, [1705 . 07936].

[63] M. Chrzaszcz, M. Drewes, T. E. Gonzalo, J. Harz, S. Krishnamurthy and C. Weniger, A frequentist analysis of three right-handed neutrinos with GAMBIT, Eur. Phys. J. C 80 (2020) 569, [1908.02302].

[64] GAMBIT Cosmology Workgroup collaboration, J. J. Renk et al., CosmoBit: A GAMBIT module for computing cosmological observables and likelihoods, JCAP 02 (2021) 022, [2009.03286].

[65] GAMBIT collaboration, G. D. Martinez, J. McKay, B. Farmer, P. Scott, E. Roebber, A. Putze et al., Comparison of statistical sampling methods with ScannerBit, the GAMBIT scanning module, Eur. Phys. J. C 77 (2017) 761, [1705.07959].

[66] GAMBIT collaboration, P. Athron et al., Global fits of GUT-scale SUSY models with GAMBIT, Eur. Phys. J. C 77 (2017) 824, [1705.07935].

[67] GAMBIT collaboration, P. Athron et al., A global fit of the MSSM with GAMBIT, Eur. Phys. J. C 77 (2017) 879, [1705.07917].

[68] GAMBIT collaboration, P. Athron et al., Combined collider constraints on neutralinos and charginos, Eur. Phys. J. C 79 (2019) 395, [1809. 02097].

[69] GAMBIT collaboration, P. Athron et al., Status of the scalar singlet dark matter model, Eur. Phys. J. C 77 (2017) 568, [1705.07931]. 
[70] P. Athron, J. M. Cornell, F. Kahlhoefer, J. Mckay, P. Scott and S. Wild, Impact of vacuum stability, perturbativity and XENONIT on global fits of $\mathbb{Z}_{2}$ and $\mathbb{Z}_{3}$ scalar singlet dark matter, Eur. Phys. J. C 78 (2018) 830, [1806.11281].

[71] GAMBIT collaboration, P. Athron et al., Thermal WIMPs and the Scale of New Physics: Global Fits of Dirac Dark Matter Effective Field Theories, 2106. 02056.

[72] S. Hoof, F. Kahlhoefer, P. Scott, C. Weniger and M. White, Axion global fits with Peccei-Quinn symmetry breaking before inflation using GAMBIT, JHEP 03 (2019) 191, [1810.07192].

[73] P. Athron et al., Global fits of axion-like particles to XENONIT and astrophysical data, JHEP 05 (2021) 159, [2007.05517].

[74] J. Bhom, M. Chrzaszcz, F. Mahmoudi, M. T. Prim, P. Scott and M. White, $A$ model-independent analysis of $b \rightarrow s \mu \mu^{-}$transitions with GAMBIT's FlavBit, 2006.03489.

[75] GAMBIT Cosmology Workgroup collaboration, P. Stöcker et al., Strengthening the bound on the mass of the lightest neutrino with terrestrial and cosmological experiments, Phys. Rev. D 103 (2021) 123508, [2009.03287].

[76] N. D. Christensen and C. Duhr, FeynRules - Feynman rules made easy, Comput. Phys. Commun. 180 (2009) 1614-1641, [0806.4194].

[77] A. Alloul, N. D. Christensen, C. Degrande, C. Duhr and B. Fuks, FeynRules 2.0 - A complete toolbox for tree-level phenomenology, Comput. Phys. Commun. 185 (2014) 2250-2300, [1310.1921].

[78] F. Staub, SARAH, 0806.0538.

[79] F. Staub, SARAH 3.2: Dirac Gauginos, UFO output, and more, Comput. Phys. Commun. 184 (2013) 1792-1809, [1207.0906].

[80] F. Staub, SARAH 4 : A tool for (not only SUSY) model builders, Comput. Phys. Commun. 185 (2014) 1773-1790, [1309.7223].

[81] A. V. Semenov, LanHEP: A Package for automatic generation of Feynman rules in gauge models, hep-ph/9608488.

[82] A. V. Semenov, LanHEP: A Package for automatic generation of Feynman rules in field theory. Version 2.0, hep-ph/0208011.

[83] A. Semenov, LanHEP: A Package for the automatic generation of Feynman rules in field theory. Version 3.0, Comput. Phys. Commun. 180 (2009) 431-454, [0805. 0555].

[84] R. Mertig, M. Bohm and A. Denner, FEYN CALC: Computer algebraic calculation of Feynman amplitudes, Comput. Phys. Commun. 64 (1991) 345-359. 
[85] J. Kublbeck, M. Bohm and A. Denner, Feyn Arts: Computer Algebraic Generation of Feynman Graphs and Amplitudes, Comput. Phys. Commun. 60 (1990) 165-180.

[86] T. Hahn, Generating Feynman diagrams and amplitudes with FeynArts 3, Comput. Phys. Commun. 140 (2001) 418-431, [hep-ph/0012260].

[87] A. Belyaev, N. D. Christensen and A. Pukhov, CalcHEP 3.4 for collider physics within and beyond the Standard Model, Comput. Phys. Commun. 184 (2013) 1729-1769, [1207.6082].

[88] G. Uhlrich, F. Mahmoudi and A. Arbey, MARTY - Modern ARtificial Theoretical phYsicist A C++ framework automating symbolic calculations Beyond the Standard Model, Comput. Phys. Commun. 264 (2021) 107928, [2011.02478].

[89] S. Bloor, T. E. Gonzalo, P. Scott, C. Chang, A. Raklev, J. E. Camargo-Molina et al., The GAMBIT Universal Model Machine: from Lagrangians to Likelihoods, 2107.00030. 